with drug treatment, non-adherence to advice by doctors, and the pressures of limited time and resources.

A point in case is the editorial by Joel A Simon on the control of hypertension in elderly people. Clinicians are instructed to treat all patients with a blood pressure of over $160 / 90 \mathrm{~mm} \mathrm{Hg}$, irrespective of age - but not to lower the pressure too far, for fear of entering the upstroke of the J shaped curve. How practical is this advice, and does it lead to demoralisation among those trying to treat their hypertensive patients?

My group practice covers a population of 14500 , is fully computerised, is a research practice of the Royal College of General Practitioners, and runs specific hypertension clinics. How do we measure up to the standards set in the editorial for lowering blood pressure? Of 771 treated patients attending for surveillance of hypertension, a fifth (160) had diastolic pressures of $>90 \mathrm{~mm} \mathrm{Hg}$ and seven tenths (536) had systolic pressures of $>140 \mathrm{~mm} \mathrm{Hg}$. Our practice is unlikely to be much different from many in Britain.

Surely it would be more appropriate for achievable targets to be discussed in an editorial, with health gains per $\mathrm{mm} \mathrm{Hg}$ fall in systolic or diastolic pressure being a more pragmatic (and, indeed, encouraging) goal for primary health care teams genuinely trying their best.

DAVID SEAMARK Honiton Group Practice, Honiton EX14 8DD

1 Simon JA. Treating hypertension: the evidence from clinical trials. $B M \mathcal{F}$ 1996;313:437-8. (24 August.)

\section{Communicating risk}

Use of standard terms is unlikely to result in standard communication

EdrToR,-Kenneth C Calman proposes a language of risk that seeks to introduce standardisation into our communication with patients about risks. ${ }^{1}$ The debate that he calls for must address three fundamental issues. Firstly, is it feasible or theoretically justifiable to translate estimates of risk derived from populations to individuals? Secondly, does current communication about risks meet patients' requirements and is it relevant to them? Thirdly, if we continue with a pragmatic approach to communication about risk, developing it in terms of language, how is this best manifested in practice?

Understanding of risk differs among epidemiologists, clinicians, and lay people. In epidemiology, risk expresses as a statistical measure the association between a characteristic and a disease in a defined population. Tension exists, however, between this perspective, which derives most closely from the "frequentist" interpretation of probability, and the lay perspective. $^{2}$ The lay perspective corresponds with the "subjectivist" interpretation of probability, reflecting the context and our expectations and observations of events. ${ }^{2}$

Clinicians may view themselves as trying to mediate between these two, using epidemiological data about groups to help individual decision making in practice. But communication about clinical risks is not straightforward, which perhaps suggests that estimates of risk derived from populations are unhelpful in the individual setting (as the subjectivist perspective holds) and that current practice in communicating risks fails to meet patients' needs. People often have an "all or nothing" perspective of harm or risk (and may therefore, for example, continue to smoke, or not take any risk with a new drug or an operation), showing that epidemiologically derived estimates of risk are often irrelevant to them. ${ }^{3}$
We still need to know more about what information about risks patients require. Perhaps our language for communicating risks should not be complicated by the uncertainty of applying population estimates to individuals, or perhaps we should continue to bridge this gap from population to individual. This is where a consistent and standardised language could help, but we must address other aspects too. Use of standard terms in the assessment of risk is unlikely to result in standard communication because the interpretation of the language of risks by patients and doctors varies ${ }^{45}$ and because of other contributions to the communication process: non-verbal elements, mental images, past experiences, and discussion about the meaning of risk to individuals. Standard terms would have to be sufficiently flexible to accommodate these contributions. Research to improve communication about risks may need to consider innovation in all these areas and identify whether patient oriented outcome measures are improved by the new processes.

ADRIAN EDWARDS Clinical fellow ROISIN PILI Professor of general practice research NIGEL STOT Professor of general practice University of Wales College of Medicine,

Department of General Practice,

Health Centre,

Maelfa,

Llanedeyrn,

Cardiff CF3 7PN

1 Calman KC. Cancer: science and society and the communication of risk. $B M F$ 1996;313:799-802. (28 September.)

2 Prior L, Bunton R, Nettleton S, Burrows R, eds. Chance and modernity: accidents as a public health problem. In: The sociology of health promotion. London: Routledge, 1995:133 44.

3 Adelsward V, Sachs L. The meaning of 6.8: numeracy and normality in health information talks. Soc Sci Med normality in heal

4 Bryant GD, Norman GR. Expressions of probability: word and numbers. N Engl F Med 1980;302:411.

5 Mazur DJ. Interpretation of graphic data by patients in a general medical clinic. $f$ Gen Intern Med 1990;5:402-5.

Risks can also be categorised as normal or abnormal

EdIToR,-Kenneth C Calman's article on science and society and the communication of risk opens a much needed debate on the public perception of risk. ${ }^{1}$ It defines many of the words used in the assessment of risk, but I would suggest that a further factor that is implicit might be added, though maybe it should be more explicit. To Calman's concept of pairs of terms (avoidable/unavoidable, justifiable/unjustifiable, etc) I would add another pair-normal abnormal. This is close to the acceptable/nonacceptable pair but not identical.

As a community, we accept some risks as normal even when their values are quite high. Examples include accidents on the road ( 1 in 8000), falling off a ladder while painting the bedroom, and cutting off a thumb with a hedge trimmer. These are accepted, albeit with some grumbling, as "part of life." Other risks, such as being infected with a virus in the course of treatment, being exposed to natural radiation, or becoming infected by a virus while swimming, are often perceived as not being part of normal life; though in the range of very low to negligible risks, they are regarded as unacceptable. Some others, such as the risk of being mugged in the street, lie somewhere between and are usually ignored except after a particularly well publicised event.

Whether a particular risk is regarded as normal or abnormal varies greatly over time. For example, we no longer expect a man with a red flag to walk in front of a car, and this reflects how well the general public comes to terms with different examples. Assessment of risks has to go closely with education if we are to manage risk sensibly, and, because it takes time to come to terms with new factors, such education takes longer than we would prefer. Without it, perception is uninformed and the response volatile. As a result, as Calman says, we face spending undue amounts of money on abating negligible risks.

DICK MADELEY

Public Health Laboratory,

Professor of clinical virology

Institute of Pathology,

Newcastle General Hospital,

Newcastle upon Tyne NE4 6BE

1 Calman KC. Cancer: science and society and the communication of risk. $B M Y$ 1996;313:799-802. (28 September.)

\section{Prescribing incentive scheme for non-fundholding general practices}

Tailoring a scheme to individual practices is more effective

EDITOR,-D N Bateman and colleagues' paper adds to the evidence that incentives to general practitioners are an important component of a strategy to change doctors' prescribing patterns. ${ }^{1}$ It is interesting to compare the results of the scheme in the Northern region in England, described by Bateman and colleagues, with those of the rational prescribing payment scheme in Lothian, Scotland. Both schemes contained financial incentives for non-fundholding practices and led to savings in prescribing. The Lothian scheme consisted of three targets for practices: to be within budget, to achieve a rate of prescribing of generic drugs of over $56 \%$, and to achieve a quality target. Table 1-Comparison of main features of schemes in
Northern region and Lothian. Figures are numbers Northern region and Lothian. Figures are
(percentages) except where stated otherwise

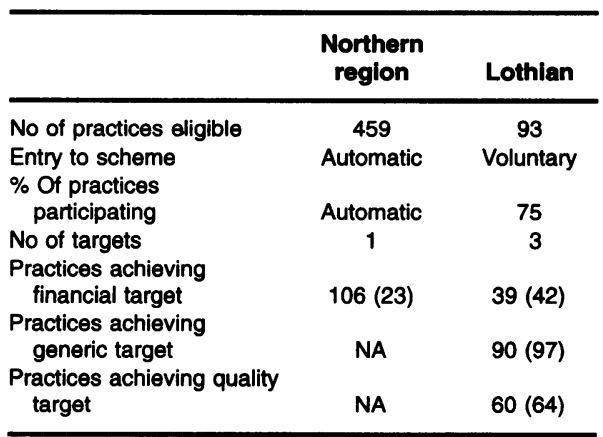

NA $=$ Not applicable

The main difference between the schemes was the way they were applied. In Lothian methods that have been shown to be effective in changing doctors' behaviour were used. These have been described by $\mathrm{Grol}^{2} \mathrm{He}$ recommends the use of face to face education, assessment, and feedback by well respected peers, combined with practical support (including financial stimuli), as a particularly effective means of improving the quality of care. He has shown that coercive controlling methods can have a negative effect at one end of the spectrum and facilitating educational methods a positive effect at the other. An essential part of Lothian's scheme included a practice meeting with the health board's medical prescribing adviser to set the targets and allow discussion.

The success of these schemes could be measured by looking at the number of practices taking part (uptake) or the number achieving their targets, or both. Interestingly, the results are 
different, probably because of the way the schemes were applied. It is difficult to know how many of the practices participated actively in the Northern region's scheme because entry to the scheme was automatic; the uptake in Lothian was $75 \%$. Table 1 shows the proportion of practices in both regions that achieved their financial target and the proportions in Lothian that achieved the target for generic prescribing and the quality target. The quality target produced 33 audits of prescribing and 12 quality standards for prescribing at practice level.

The disadvantage of applying Lothian's scheme is that it is time consuming for both practices and prescribing advisers, but the results suggest that tailoring a scheme to individual practices is an effective strategy.

PHILIP RUTLEDGE Senior medical adviser

Lothian Health,

Edinburgh EG8 9RS

I Bateman DN, Campbell M, Donaldson LJ, Roberts SJ, Smith JM. A prescribing incentive scheme for non-fundholding general practices: an observational study. BM9 general practices: an obs:

2 Grol $R$. Implementing guidelines in general practice care. Quality in Health Care 1992;1:184-91.

\section{Reduction in prescribing cannot}

\section{confidently be attributed to scheme}

EDrror,-D N Bateman and colleagues set out to examine the effects of a financial incentive scheme on prescribing among non-fundholding general practitioners. We are not convinced, however, that they have shown that these general practitioners responded to such incentives in a manner similar to that of fundholding practices, as the authors claim.

Firstly, from a purely descriptive viewpoint, the proportion of practices achieving the target reduction was relatively modest $(23 \%$ of all practices). More importantly, however, there are no control data either from practices outside the scheme or in the form of historical trend data from the practices in the scheme. As the authors acknowledge in their discussion-but ignore in their abstract - this means that causal attribution of the reduction in prescribing to the incentive scheme is not possible with confidence. Other, secular, trends may have been responsible. Correcting this important deficit is a question not merely of undertaking further analysis, as the authors suggest, but of acquiring new data and considerably altering the design of this simple "before and after" quasiexperiment.

The second flaw is that all the comparisons between the so called achieving and nonachieving practices are based not on changes in prescribing behaviour but on simple cross sectional data. There is no way of knowing whether the significantly lower prescribing costs for, for example, inhaled steroids and antidepressants in achieving practices were of long standing or developed for the first time during the study.

In summary, having failed to show whether any change was attributable to the scheme and to identify in which drug categories any changes (attributable or otherwise) occurred, this study provides no valid evidence of whether incentive schemes alter prescribing costs and quality.

\section{ELIZABETH ROBINSON}

Avon Health Authority, Medical adviser Bristol BS2 BEE

IAN HARVEY

Consultant senior lecturer in public health medicine Department of Social Medicine,

Bristol BS8 2PR

1 Bateman DN, Campbell M, Donaldson LJ, Roberts SJ, Smith JM. A prescribing incentive scheme for non-fundholding general practices: an observational study. BMף general practices: an obst
1996;313:535-8. (31 August.)

\section{Authors' reply}

EDrToR,-The proportion of practices achieving financial targets will depend on the target set and whether practices are specifically selected. Prescribing regulations in England and Wales now place a statutory obligation on health authorities to operate incentive schemes that are comprehensive and include all non-fundholding practices in a manner similar to the scheme in our study. ${ }^{12}$

We agree with Philip Rutledge that incentive schemes seem to have some effect in limiting the growth in prescribing costs. Our approach produced worthwhile gain without imposing the demands on advisers' and general practitioners' time that are associated with targeted schemes such as the one in Lothian.

As was clearly stated in the title of our paper, our study was an observational one. Studies of prescribing behaviour may be affected by secular trends and selection bias. It is extremely rare in observational studies to show clear cause and effect, and Elizabeth Robinson and Ian Harvey's criticisms thus seem in part to be based on a misapprehension. Randomised controlled studies are clearly to be preferred, but operationa constraints made this impossible. Comparison of non-randomised groups and trends over time and direct comparison with practitioners outside the region are all potentially subject to confounding. All studies of the prescribing behaviour of fundholding practitioners are non-randomised and subject to the same criticisms.

Robinson and Harvey suggest that the changes that we observed reflected existing cross sectional differences. We acknowledged this in our discussion, but the other research that we quoted clearly indicates that practices made conscious decisions with respect to the scheme. ${ }^{3}$ The region has continued to show good financial performance of non-fundholders relative to the rest of England, which suggests that changes in prescribing have been sustained.

We remain puzzled why Robinson and Harvey adopt such a negative attitude to our report, in which we measured prescribing behaviour in the context of a financial incentive scheme that offered practitioners a modest reward for efforts to promote cost effective prescribing.

D N BATEMAN

Medical directo

M CAMPBELI

Manager, prescribing unit $S$ J ROBERTS

Lecturer in medical statistic

J M SMTTH

Regional pharmaceutical advise

Regional Drug and Therapeutics Centre,

Wolfson Unit,

Newcastle upon Tyne NE2 4HH

L J DONALDSON

Department of Epidemiology and Public Health

University of Newcastle,

Newcastle upon Tyne NE2 4HH

1 Statutory instrument 1995 No 692. National Health Service (functions of family health services authorities) (prescribin incentive schemes) regulations 1995. London: HMSO, 1995. 2 incentive schemes) regulations 1995. London: HMSO, 1995. NHSE, 1995. (FHSL (95)20.)

3 Eccles MP, Soutter J, Bateman DN, Campbell M, Smith JM Influences on prescribing in non-fundholding general practices. Br $\mathcal{O}$ Gen Pract 1996;46:287-90.

\section{Side effects of tamoxifen are distressing and common}

EDIToR,-The letters by $R$ D Bulbrook and Michael Baum and Jack Cuzick reflect the medical interest in the long term risks of prophylactic tamoxifen in ostensibly well women who are at risk of breast cancer as well as in the prevention or delay of relapse after surgery for symptomatic disease. $^{12}$ Our research shows that the side effects associated with tamoxifen are neither "overstated" nor "anecdotal."

We carried out a questionnaire study of women with advanced breast cancer and were surprised by the frequency and severity of side effects due to this drug as well as other to hormonal agents. The level of problems was very different from the toxicities associated with most chemotherapies but was nevertheless substantial.

We collected prospective information on side effects in 161 patients taking adjuvant tamoxifen and 38 controls matched for postmenopausal status. When we compared symptoms between the two groups we found significant differences $(\mathrm{P}<0.01)$ for hot flushes (75 (47\%) patients $v 6$ (16\%) controls), weight gain (71 (44\%) v $7(18 \%)$ respectively), and vaginal discharge (45 $(28 \%) v 1(3 \%))$. Patients often described the symptoms as "worrying."

The lesson from this is that one should never underestimate the potential for well tolerated drugs to cause problems. The drug datasheets are often more worrying than reassuring. The severity of adverse effects is not the only consideration: the side effects identified in this study are for the most part not dangerous but nevertheless distressing and common. Accurate information about side effects must be disclosed to patients.

MRIT RAY Medical student ROBERT C F LEONARD Consultant medical oncologist

Department of Clinical Oncology,

Western General Hospital,

Edinburgh EH4 2XU

1 Bulbrook RD. Adjuvant treatment with tamoxifen. $B M \mathcal{J}$ 1996;313:493-4. (24 August.)

2 Baum M, Cuzick J. Adjuvant treatment with tamoxifen. $B M f$ 1996;312:1036. (20 April.)

\section{Value of milk tokens for breast feeding mothers should be increased}

EDITOR,- In the deprived housing estates on the periphery of Glasgow roughly $7 \%$ of babies are breast fed at the end of the first week of life. Much research has related infant feeding to maternal background and socioeconomic status, ${ }^{12}$ and, despite promotional efforts, breast feeding continues to decrease in these areas.

Families receiving income support are eligible for milk tokens, which can be exchanged for 7 pints (4 litres) of milk a week by breast feeding mothers or for one $900 \mathrm{~g}$ tin of infant formula a week by mothers who bottle feed their baby. The financial value of the milk token is about $£ 2.45$ for breast feeding mothers, compared with $£ 6.95$ for mothers who bottle feed. It has been suggested that milk tokens may discourage breast feeding. ${ }^{3}$

Information was collected from women attending for their first antenatal appointment who lived in an area of urban deprivation. (The area's deprivation category score was 7; this measure of deprivation in small areas is based on the percentage of households with no car, overcrowding, male unemployment, and low social class, as defined by Carstairs and Morris. ${ }^{4}$ ) Altogether 704 women completed a questionnaire asking whether they intended to breast feed or bottle feed their baby and examining influencing factors. They were asked whether they received milk tokens and whether this affected their choice of feeding.

Most women stated that their choice was unaffected by the receipt of tokens, though a few remarked that they would not be able to afford to bottle feed if they did not receive tokens. Statistical analysis of the data in table 1 shows that 